\title{
Anesthesia for non-obstetric surgery during pregnancy in a tertiary referral center: a 16-year retrospective, matched case-control, cohort study
}

\author{
S. Devroe, ${ }^{\mathrm{a}, \mathrm{b}}$ T. Bleeser, ${ }^{\mathrm{a}}$ M. Van de Velde, ${ }^{\mathrm{a}, \mathrm{b}}$ L. Verbrugge, ${ }^{\mathrm{a}}$ F. De Buck, ${ }^{\mathrm{a}}$ J. Deprest, ${ }^{\mathrm{c}, \mathrm{d}}$ \\ R. Devlieger, ${ }^{\mathrm{c}, \mathrm{d}}$ S. Rex ${ }^{\mathrm{a}, \mathrm{b}}$ \\ ${ }^{a}$ Department of Anesthesiology, University Hospitals of the KU Leuven, Herestraat 49, 3000 Leuven, Belgium \\ ${ }^{\mathrm{b}}$ Department of Cardiovascular Sciences, KU Leuven, Herestraat 49, 3000 Leuven, Belgium \\ ${ }^{\mathrm{c}}$ Department of Obstetrics and Gynecology, University Hospitals of the KU Leuven, Herestraat 49, 3000 Leuven, \\ Belgium \\ ${ }^{\mathrm{d}}$ Department of Development and Regeneration, KU Leuven, Herestraat 49, 3000 Leuven, Belgium
}

\begin{abstract}
Introduction: This retrospective, matched case-control cohort study describes the incidence, indications, anesthesia techniques and outcomes of pregnancies complicated by surgery in a single tertiary-referral hospital.

Methods: Retrospective review of the hospital records of 171 patients who had non-obstetric surgery in the current pregnancy, between 2001 and 2016. Pregnancy outcomes of these women were firstly compared with all contemporary non-exposed patients $(\mathrm{n}=35411)$, and secondly with 684 non-exposed control patients, matched for age, time of delivery and parity.

Results: The incidence of non-obstetric surgery during pregnancy was $0.48 \%$, mostly performed during the second trimester ( $44 \%)$ and under general anesthesia (81\%). Intra-abdominal surgery $(44 \%)$ was the most commonly performed procedure, predominantly using laparoscopy (79\%). Women undergoing surgery delivered earlier and more frequently preterm $(25 \%$ vs. $17 \%, P=0.018)$; and birth weight was significantly lower [median (95\% CI) 3.16 (3.06 to 3.26$)$ vs. 3.27 (3.22 to 3.32$) \mathrm{kg}, P=0.044$ ]. When surgery was performed under general anesthesia, low birth weight was more frequent $(22 \%$ vs. $6 \%, P=0.046)$. Overall pregnancy outcomes were neither influenced by trimester nor location (intra- vs. extra-abdominal) of surgery. However, preterm birth rate secondary to surgery was higher for interventions during the third trimester, compared with other trimesters $(10 \%$ vs. $0, P<0.001)$.

Conclusion: Pregnant women who underwent surgery delivered preterm more frequently and their babies had lower birth weights. Laparoscopic surgery did not increase the incidence of adverse pregnancy outcomes. General anesthesia was associated with low birth weight. Whether these associations suggest causation or reflect the severity of the underlying condition remains speculative. (c) 2019 Elsevier Ltd. All rights reserved.
\end{abstract}

Keywords: Non-obstetric surgery, incidence; Anesthesia; Pregnancy outcome; Preterm delivery; Low birth weight; Caesarean section

\section{Introduction}

The need for a non-obstetric surgical or invasive intervention requiring anesthesia may occur at any stage of pregnancy. Previous studies of pregnant women have reported that $0.2-2.2 \%$ undergo an operative intervention unrelated to their pregnancy. ${ }^{1-5}$ Unfortunately, scientific evidence about this subject is sparse and clinical management is primarily driven by traditional belief and personal preferences. ${ }^{2,3}$

Accepted January 2019

Correspondence to: S. Devroe, Department of Anesthesiology,

Herestraat 49, 3000 Leuven, Belgium.

E-mail address: sarah.devroe@uzleuven.be
Although the unborn child is an "innocent bystander" in non-obstetric procedures, perinatal outcomes have been shown to be significantly compromised. A study from the United Kingdom reviewed 6.5 million pregnancies and found an incidence for non-obstetric surgery of $0.9 \%$ and an additional risk for preterm delivery $(3.2 \%)$, low birth weight $(2.6 \%)$ and cesarean delivery $(\mathrm{CD})(4.0 \%) .^{5}$

We performed a retrospective analysis of all non-obstetric surgical procedures in pregnant women performed in a single center over a 16-year period. The incidence, indications, type and timing of surgery, anesthetic techniques, and obstetric and neonatal 
outcomes were assessed. Pregnancy outcome, both obstetric and neonatal, was compared firstly to the entire cohort of women giving birth in our hospital during the same period, and secondly to a matched case-control cohort consisting of women who gave birth at the same age, within the same month and at the same parity as the non-obstetric surgical cases. To the best of our knowledge no matched case-control data are available on this subject, which makes our study design unique.

\section{Methods}

The study was approved by the local ethics committee (S60949, Commissie Medische Ethiek, Universitaire Ziekenhuizen Leuven, Belgium, December 20, 2017). The University Hospitals of Leuven is a tertiary obstetric referral center with 2200-2500 deliveries each year. The Department of Obstetrics is a local, national and international referral center for severe fetal and maternal pathology, but also offers low-risk obstetric services. The population, therefore, consists of a mixture of low and high-risk patients, but with an over-representation of high-risk patients (about 50\%) compared to the general obstetric population.

A retrospective chart review of the electronic patient files of all women who delivered at the University Hospitals of Leuven over a 16-year period (January 1, 2001 to December 31, 2016) was performed. Women who underwent surgery during pregnancy were identified by a search algorithm linking delivery to an anesthesia contact that took place within 280 days before delivery. Women who underwent obstetric procedures (e.g. cervical cerclage) or fetal surgical interventions were excluded.

The following data were retrieved from the hospital information system: demographic data, procedural characteristics (type of intervention, duration, timing of the intervention in relation to the duration of gestation), type of anesthesia (general (GA) vs. locoregional (RA)) and pregnancy outcome including week of gestation at delivery, birth weight, vaginal or $\mathrm{CD}$, and interval between surgery and delivery.

Pregnancy outcomes of these patients were compared with contemporary controls consisting of all women giving birth in our institution over the same period who had no non-obstetric invasive interventions requiring anesthesia during pregnancy. In patients who underwent multiple interventions, only the first intervention was taken into account for pregnancy outcome statistics. For the control cohort, all relevant data were obtained from the regional study center for perinatal epidemiology (Studiecentrum voor Perinatale Epidemiologie, SPE).

In an attempt to control for known confounding factors and reduce the possible risk of bias, a matched, controlled analysis was performed. Patients were matched to controls in a 1:4 ratio using the following matching factors: age at delivery, date of delivery and parity. These factors were chosen to minimize the influence of variation in medical practice during the relatively long investigation period and the influence of age and parity on birth weight and prematurity. ${ }^{6}$

All data were analyzed using a commercially available software package (GraphPad Prism 7, GraphPad Software, La Jolla, CA, USA). Proportions were tested using the $\chi^{2}$ test. A two-sided $P$-value $<0.05$ was considered statistically significant.

For the matched case-control analysis, a linear mixed model with a random effect for each set of one case and four controls was used to compare the birth weight and gestational age between cases and controls. Incidences of preterm delivery, birth weight $<2500 \mathrm{~g}, \mathrm{CD}$ rate and choice of combined spinal-epidural/epidural anesthesia during delivery were evaluated with a conditional logistic regression. Since birth weight and gestational age were heavily left-skewed, a Box-Cox transformation was applied. Back-transformation to the original scale yields approximate medians. The $95 \%$ confidence intervals (CI) were reported for these approximate medians and for the odds ratios (OR) obtained in the conditional logistic regression models. $P$-values less than 0.05 were considered significant. No corrections for multiple testing were considered, and therefore a single $P$-value should be interpreted with caution. All analyses were performed using SAS software (SAS System for Windows v 9.4).

\section{Results}

\section{Study population and incidence of surgery}

The study flow chart is shown in Fig. 1. In total, 35612 deliveries took place during the 16-year observation period. A total of 171 women underwent 189 non-obstetric surgical interventions requiring regional or general anesthesia (intervention group); 10 patients underwent two or more surgical interventions. The overall incidence of non-obstetric surgery during gestation was $0.48 \%$, and this did not change significantly over the years (range $0.27-0.90 \%$ ). The entire control group consisted of all other women who gave birth in our hospital during the same time period ( $\mathrm{N}=35441)$. The matched control group included 684 women matched for age, parity and month of delivery.

\section{Type and timing of surgery}

Table 1 gives an overview of the 189 different nonobstetric procedures requiring anesthesia during pregnancy. The most common operations were intraabdominal $(44 \%)$, of which 36 were appendectomies and 12 were interventions for ovarian torsion, cyst or mass. The second most common indication was trauma surgery $(15 \%)$. Most of the procedures were performed 


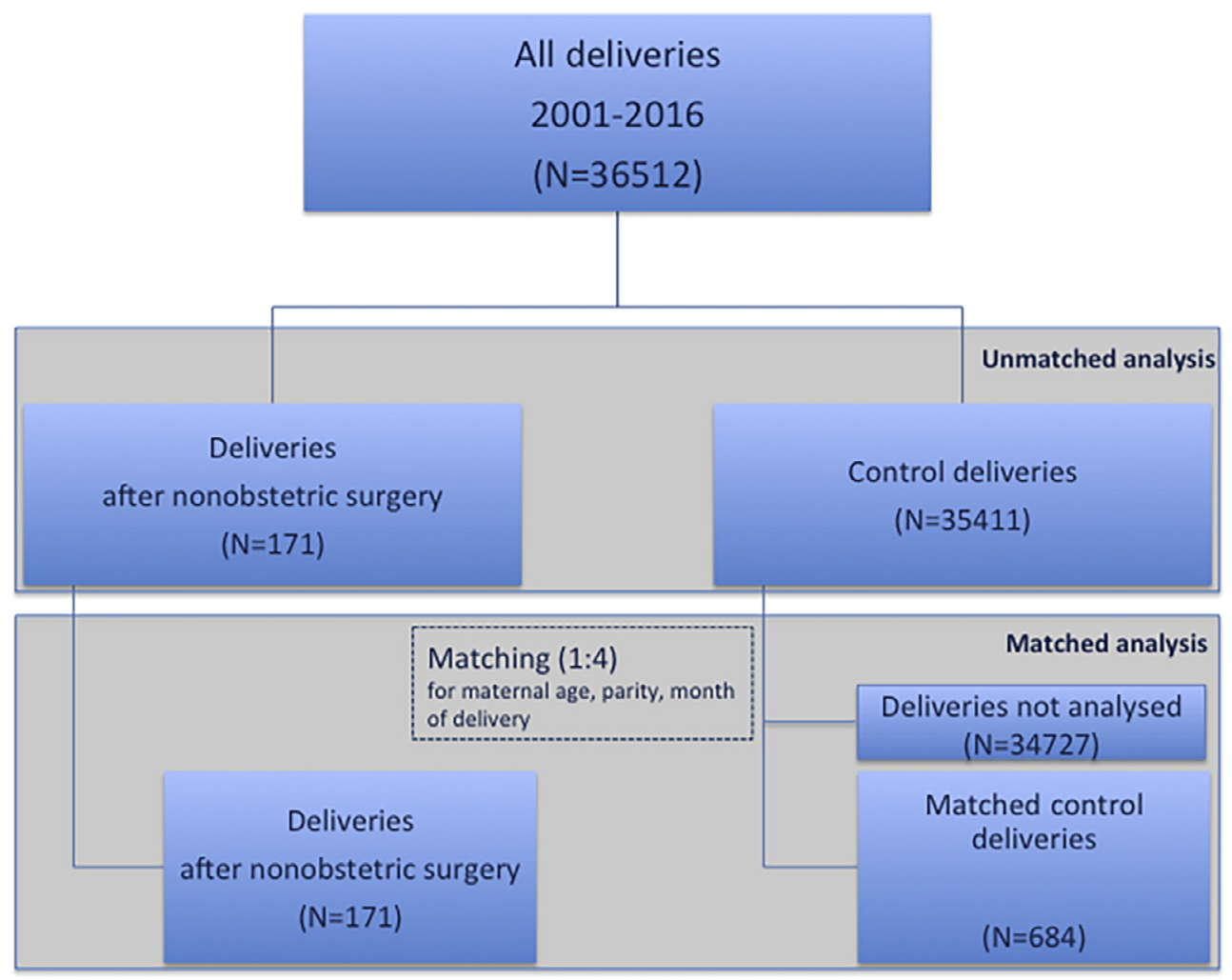

Fig. 1 Study flow chart.

during the second trimester (44\%), while 32\% were carried out in the first trimester and $24 \%$ in the third trimester.

\section{Anaesthetic technique}

General anesthesia was used in $81 \%$ of cases and RA, (with or without additional sedation) was used in $19 \%$ of cases. Loco-regional anesthesia was mostly administered for orthopedic or trauma surgery on body extremities and in reconstructive surgery (Table 1).

\section{Pregnancy outcome}

\section{Cases versus entire birth cohort}

Significantly more women in the intervention group delivered preterm $(<37$ weeks) than in the control group $(P<0.001)$ (Table 2). There was no difference in the incidence of low birth weight neonates $(P=0.289)$. More women from the intervention group underwent $C D$ ( $P=0.01)$.

There were three $(1.75 \%)$ terminations of pregnancy due to major congenital birth defects in the intervention group. One patient had undergone resection of an atrial myxoma on cardio-pulmonary bypass at 10 weeks-ofgestation and pregnancy termination took place at 31 weeks-of-gestation due to caudal dysgenesis and intrauterine growth restriction: one patient underwent appendectomy at 16 weeks-of-gestation and pregnancy termination took place at 21 weeks-of-gestation due to fetal hypoplastic left heart syndrome: and one patient underwent appendectomy at 26 weeks-of-gestation and pregnancy termination at 28 weeks-of-gestation due to hydrops fetalis of unknown origin. In the control group, there were $535(1.5 \%)$ fetal losses (spontaneous or induced for major congenital defects). The incidences between groups were not significantly different between groups $(P=0.74)$.

\section{Cases versus matched controls}

Cases delivered earlier ( 38.59 vs 38.95 weeks; $P=0.02$ ) and preterm more frequently $(24.6 \%$ versus $16.7 \%$; OR 1.63; $P=0.018$ ) than their matched controls. Birth weight was also lower in the interventional group (3.16 kg vs $3.27 \mathrm{~kg} ; P=0.04$ ), but the incidence of low birth weight $(<2500 \mathrm{~g})$ did not differ significantly (18.1\% vs. $13.9 \%$; OR $1.36 ; P=0.176)$ from the matched cohort. There was no difference in the rate of $\mathrm{CD}$ between cases and the control group.

We did not attempt to determine the incidence of fetal loss in this matched analysis because the register in which we searched for the controls only included births and fetal losses after 20 weeks-of-gestation.

\section{Association of pregnancy outcome with timing of surgery}

Table 3 shows pregnancy outcome according to the trimester in which surgery was performed. A comparison 
Table 1 Procedures that were performed during pregnancy

\begin{tabular}{|c|c|c|c|c|}
\hline Surgery & & & General anesthesia & Regional anesthesia \\
\hline Intra-abdominal surgery & 84 & $(44)$ & 83 & 1 \\
\hline \multicolumn{5}{|l|}{ Appendectomy } \\
\hline Laparoscopic & 29 & & 29 & 0 \\
\hline Open & 7 & & 7 & 0 \\
\hline \multicolumn{5}{|l|}{ Ovarian detorsion/mass resection } \\
\hline Laparoscopic & 10 & & 10 & 0 \\
\hline Open & 2 & & 2 & 0 \\
\hline \multicolumn{5}{|l|}{ Reduction of internal herniation } \\
\hline Laparoscopic & 5 & & 5 & 0 \\
\hline Open & 1 & & 1 & 0 \\
\hline \multicolumn{5}{|l|}{ Other laparoscopic procedures } \\
\hline Explorative laparoscopy & 11 & & 11 & 0 \\
\hline Cholecystectomy & 4 & & 4 & 0 \\
\hline Appendectomy + adnexectomy & 1 & & 1 & 0 \\
\hline Removal gastric band & 1 & & 1 & 0 \\
\hline Gastrectomy & 1 & & 1 & 0 \\
\hline Tubectomy & 1 & & 1 & 0 \\
\hline Removal mesentery & 1 & & 1 & 0 \\
\hline Sigmoid resection & 1 & & 1 & 0 \\
\hline Intra-abdominal abscess drainage & 1 & & 1 & 0 \\
\hline
\end{tabular}

Other open/laparotomy procedures

Explorative laparotomy

Right hemi colectomy

Hartmann procedure

Pelvic lymphadenectomy

Umbilical hernia repair

Debridements (wounds/keloid/burns/toenail)

Osteosynthesis upper limb

Osteosynthesis lower limb

Arthroscopy lower limb

Urologic procedures

Ureterorenoscopy

Cystoscopy

Nephrectomy

Oncological surgery

Breast tumor resection

Axillary lymphadenectomy

Cervical lymphadenectomy

Placement Hickmann catheter

Partial glossectomy + lymphadenectomy

\section{Others}

Dental procedures

Thyroidectomy

FESS and nasal septum correction

Varicectomy

Breast abscess drainage

Jaw abscess drainage

Proctology

Anal and rectal procedures

Gynaecological surgery

Bartholin cyst marsupialisation

Cervix surgery
29

11

4
1
1
1
1
29
11
9
5
4
16
14
1

15

11

1

1

1

1

11

5

2

1

1

1

1

11

11

(6)

(5)

(8)

(8)

(6)

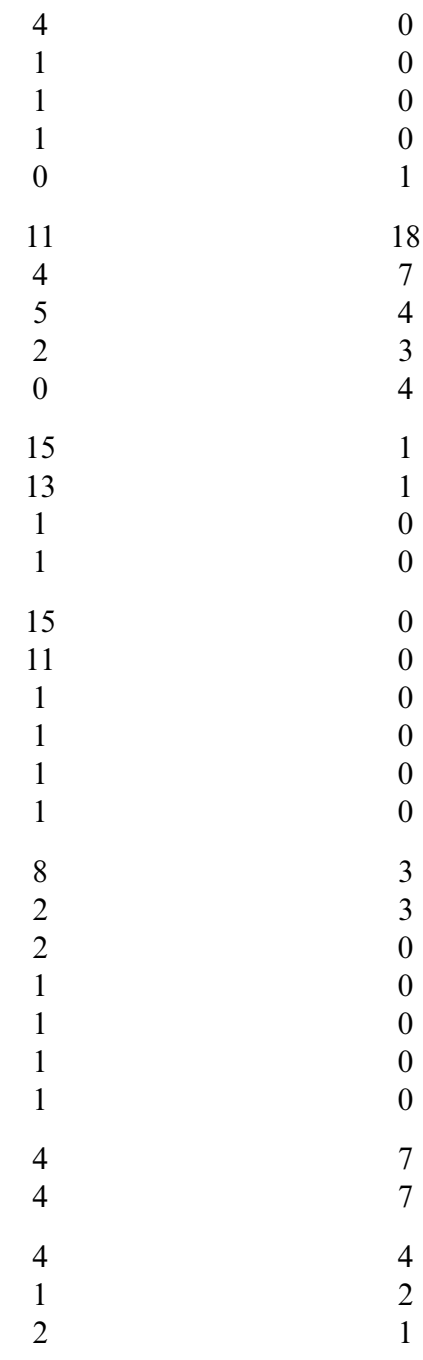




\begin{tabular}{|c|c|c|c|c|}
\hline Condylomata vaporization & 2 & & 0 & 2 \\
\hline Vulvoplasty & 1 & & 1 & 0 \\
\hline Neurosurgery & 8 & (4) & 7 & 1 \\
\hline Micro-discectomy & 3 & & 3 & 0 \\
\hline Trepanation & 2 & & 2 & 0 \\
\hline Shunt placement & 2 & & 2 & 0 \\
\hline Release carpal tunnel syndrome & 1 & & 0 & 1 \\
\hline Cardiac interventions & 6 & (3) & 6 & 0 \\
\hline Interventional cardiology & 4 & & 4 & 0 \\
\hline Open cardiac surgery & 2 & & 2 & 0 \\
\hline TOTAL & 189 & $(100)$ & $153(81)$ & $36(19)$ \\
\hline
\end{tabular}

FESS: functional endoscopic sinus surgery. Percentages of the whole are indicated in brackets.

of surgeries performed in the first, second or third trimester did not reveal a statistically significant difference in the number of preterm deliveries, incidence of low birth weight or CDs. However, the third trimester was found to be associated with the highest risk for preterm birth secondary to a surgical procedure (defined as a birth up to two weeks after the procedure). ${ }^{3}$ Only 10 $(5.85 \%)$ patients delivered within two weeks of their intervention. All of them underwent surgery in the third trimester of pregnancy $(P<0.001$ vs. trimesters one and two) with six of the deliveries being preterm $(P<0.001$ vs. trimesters one and two).

\section{Association of pregnancy outcome with the location of surgery}

Table 4 compares pregnancy outcomes stratified by operative site. A total of 189 procedures were identified in 171 women: 84 intra-abdominal and 105 extraabdominal procedures. No differences between intraand extra-abdominal surgeries were observed for the incidence of premature delivery, low birth weight, the rate of $\mathrm{CD}$ or the timing of surgery in relation to gestational age, and the interval between surgery and delivery.

Most of the abdominal surgical interventions were laparoscopic procedures $(79 \%)$ and did not result in a greater number of adverse pregnancy outcomes when compared to open procedures (data not shown).

\section{Association of pregnancy outcome with the mode of anesthesia}

In patients given GA, significantly more neonates had a low birth weight when compared to patients receiving RA. The rates of preterm or CD did not differ between the anesthesia techniques (Table 5).

\section{Discussion}

In 35612 women who delivered over a 16-year period in a single Belgian tertiary obstetric referral center, the incidence of non-obstetric invasive interventions during pregnancy was $0.48 \%$. This represents approximately $0.02 \%$ of all anesthetics administered in our center during that period. The most common procedure was abdominal surgery, most frequently carried out in the second trimester and under GA. Women undergoing invasive non-obstetric procedures requiring anesthesia when pregnant delivered earlier and more frequently when preterm; and the birth weight of the baby was significantly lower. The incidence of low birth weight was higher in women delivering after interventions performed under GA (either mandated by the intervention or chosen by the anesthesiologist).

The number of non-obstetric interventional procedures during pregnancy requiring anesthesia is low. Previous reports describe incidences of 0.2 up to $2.2 \%{ }^{3,5,7,8}$ However, some of these reports did not distinguish between obstetric and non-obstetric indications ${ }^{3}$ or even included cervical cerclage. ${ }^{8}$

Preterm delivery was the most common complication in our study population. This confirms earlier observations in which abdominal surgery during pregnancy was associated with a higher risk of preterm labor., It remains uncertain whether the increased risk of preterm delivery can be attributed to the procedure or rather to the underlying condition necessitating the intervention. Nevertheless, even late preterm birth (as seen in our study and defined as birth between 34 and 37 weeks) is associated with higher risk of morbidity and mortality in the first years of life, and is a major risk factor for the development of deficits in cognitive functioning and lower educational attainment. Individuals born preterm have higher rates of attention deficit disorder, autistic spectrum disorder and schizophrenia. ${ }^{10,11}$

In common with previous reports, we found that birth weight was significantly lower in the group of patients that underwent surgery than in the group that did not. ${ }^{2,3,12,5}$ Unlike in other series, ${ }^{5}$ this did not result in a higher frequency of low birth-weight babies $(<2500 \mathrm{~g})$. This may be explained by the fact that in tertiary referral institutions a high "background" risk for low birth weight deliveries is anticipated. In both our intervention and in our matched and entire control 


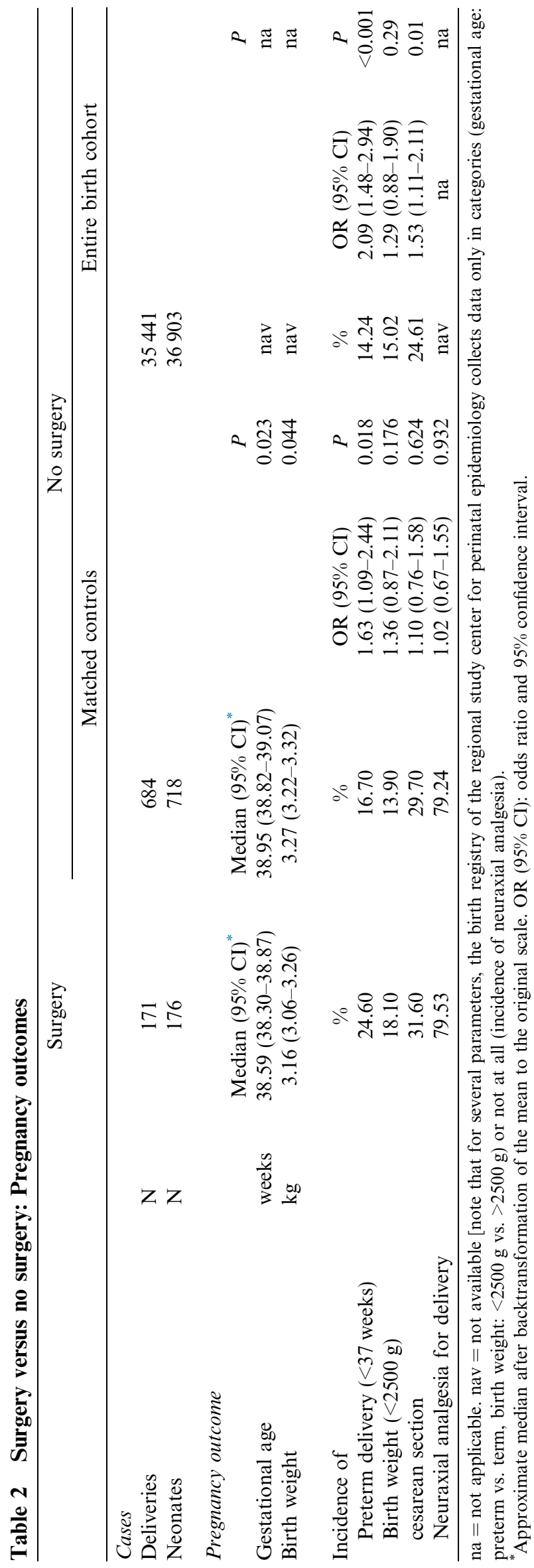

groups, there was a higher incidence of low birth weight than in the average regional population $(18 \%, 14 \%, 15 \%$ and $7 \%$ respectively).

Mothers having non-obstetric invasive procedures during the index pregnancy had higher rates of CD when compared with the entire group of controls, a finding described previously. ${ }^{12,13}$ This difference in outcome could not be confirmed by the matched case-control analysis, and highlights the importance of controlling for confounders by using a matched case-control analysis.

Previous reports have shown that rates of maternal and fetal mortality are low after interventions during pregnancy. ${ }^{3,4}$ In the present series there was no maternal morbidity, or mortality, and there were no in utero deaths. We identified three terminations of pregnancy resulting in fetal loss in the surgery group, an incidence that did not differ from the entire control group.

The most frequently performed procedures were abdominal interventions, principally appendectomies and interventions for adnexial pathology. Interestingly, there were more interventions during pregnancy related to complications after bariatric surgery. The indication for the majority of these procedures was internal herniation, which is a known complication after Roux-en $\mathrm{Y}$ gastric bypass, typically presents during the third trimester of pregnancy and is associated with adverse maternal and fetal outcomes. ${ }^{14}$

In contrast to previous reports, ${ }^{3,15}$ we found no difference in outcome between abdominal and extraabdominal surgery. This might be explained by the fact that we reported more recent data, reflecting the trend towards the predominant use of laparoscopic techniques in intra-abdominal surgery (in our series $79 \%$ ). While pregnancy was initially considered a contraindication for laparoscopic techniques, recent reports have demonstrated laparoscopic techniques during pregnancy are at least equally safe as open procedures with respect to pregnancy outcome (preterm delivery, birth weight and rate of $\mathrm{CD}$ ) and may even offer benefits (e.g. faster recovery). ${ }^{16,17}$ In our series laparoscopic procedures did not result in more adverse pregnancy outcomes compared to open procedures.

Most procedures were performed during the second trimester, in common with previous reports. ${ }^{3,8}$ It has long been assumed that surgery during the second trimester is the safest period during pregnancy, since by this stage organogenesis is virtually complete, the risk of preterm labor and delivery is small and the risk of neurotoxicity to the immature brain is most probably lower than after the onset of rapid brain growth during the first trimester. ${ }^{3,18,19}$ The lowest rate of preterm birth occurs when interventions are performed during the second trimester. ${ }^{3}$ In this study, we found no association between the overall incidence of preterm birth and the trimester in which the interventions were performed. 
Table 3 Trimester of surgery vs. pregnancy outcome

\begin{tabular}{|c|c|c|c|c|c|c|c|}
\hline \multirow[b]{2}{*}{ Surgical procedure } & \multicolumn{2}{|c|}{ First trimester } & \multicolumn{2}{|c|}{ Second trimester } & \multicolumn{2}{|c|}{ Third trimester } & \multirow[t]{2}{*}{$P$-value } \\
\hline & 54 & $(32)$ & 75 & $(44)$ & 42 & $(24)$ & \\
\hline \multicolumn{8}{|l|}{ Pregnancy outcome } \\
\hline Preterm delivery $(<37$ weeks) & 9 & (17) & 24 & $(32)$ & 11 & $(26)$ & 0.14 \\
\hline Birth weight $(<2500 \mathrm{~g})$ & 11 & $(20)$ & 13 & (17) & 8 & (19) & 0.92 \\
\hline Cesarean section & 16 & (27) & 26 & (35) & 15 & (36) & 0.78 \\
\hline
\end{tabular}

Data are presented as absolute number (percentage of the whole cohort).

Table 4 Intra-abdominal versus extra-abdominal surgery: surgery characteristics and pregnancy outcome

\begin{tabular}{|c|c|c|c|c|c|}
\hline \multirow[b]{2}{*}{ Interventions } & \multicolumn{2}{|c|}{$\begin{array}{c}\text { Intra-abdominal } \\
\text { surgery }\end{array}$} & \multicolumn{2}{|c|}{$\begin{array}{c}\text { Extra-abdominal } \\
\text { surgery }\end{array}$} & \multirow[t]{2}{*}{$P$-value } \\
\hline & 82 & $(48)$ & 89 & $(52)$ & \\
\hline \multicolumn{6}{|l|}{ Surgery-related data } \\
\hline Gestational week at surgery & 18 & {$[4-34]$} & 20 & {$[3-38]$} & 0.35 \\
\hline Duration of surgery $(\mathrm{min})$ & 75 & {$[30-120]$} & 60 & {$[15-360]$} & 0.05 \\
\hline Interval surgery-delivery (weeks) & 20 & {$[0-37]$} & 18 & {$[1-37]$} & 0.24 \\
\hline \multicolumn{6}{|l|}{ Pregnancy outcome } \\
\hline Preterm delivery (<37 weeks) & 17 & $(21)$ & 27 & $(30)$ & 0.17 \\
\hline Birth weight $<2500 \mathrm{~g}$ & 16 & $(20)$ & 15 & (17) & 0.69 \\
\hline Cesarean section & 26 & $(32)$ & 31 & (35) & 0.75 \\
\hline
\end{tabular}

Surgery related data are presented as mean [range]. Data of birth outcome are presented as absolute numbers [n, with the percentage (\%) of the whole].

Table 5 Mode of anesthesia versus pregnancy outcome

\begin{tabular}{lccc}
\hline & General anesthesia & Regional anesthesia & $P$-value \\
\hline Cases & $139(81)$ & $32(19)$ & \\
Pregnancy outcome & & $5(16)$ & 0.001 \\
Preterm delivery $(<37$ weeks) & $39(28)$ & $2(6)$ & 0.18 \\
Birth weight $(<2500 \mathrm{~g})$ & $30(22)$ & $9(28)$ & 0.046 \\
Cesarean section & $48(35)$ & 0.54 \\
\hline
\end{tabular}

Data are presented as absolute number (percentage of the whole cohort).

However, the incidence of preterm birth secondary to a surgical procedure (defined as a birth two weeks after the procedure $)^{3}$ was highest for procedures performed during the third trimester. These findings may support the longstanding belief that semi-urgent surgical procedures should be performed in the second rather than the third trimester, to avoid a preterm birth secondary to an invasive procedure.

Most invasive procedures were performed under GA. General anesthesia was associated with a higher number of low birth weight neonates, which confirms previous findings. ${ }^{3}$ Although an association between GA and lower birth weight was found, it may not be causal. For example, it has been shown that bariatric surgery is associated with lower birth weight. ${ }^{20}$ The choice of a particular anesthetic technique may depend on the preference of the anesthesiologists or patients (for example, GA versus RA for surgery on extremities), but in most circumstances the mode of anesthesia is mandated by the nature of the intervention itself. The need for GA should probably be considered a surrogate parameter for the severity of the underlying disease necessitating an intervention, and for the risk associated with that particular intervention. In our population, only a minority of procedures was performed under RA, resulting in insufficient statistical power to establish an independent association between mode of anesthesia and outcomes.

Nevertheless, based on the results of our study and considering the ongoing discussion about anesthesiarelated neurodevelopmental neurotoxicity (and the recommendation to avoid GAs during the third trimester of pregnancy, ${ }^{9}$ we at least advise giving consideration to the use of RA whenever possible. ${ }^{21}$

The study is subject to numerous limitations. First, despite including a matched control group, the retrospective nature of the study still limits the ability to correct for known and in particular unknown confounding variables affecting pregnancy outcomes. Second, data 
on early miscarriages ( $<12$ weeks-of-pregnancy) were not available. Since our data acquisition was based on delivery data (which in our delivery ward only registers deliveries from 12 weeks-of-gestation), we cannot exclude the possibility that interventions during early pregnancy that resulted in first trimester miscarriages were not included in our series. Third, patients who underwent an invasive procedure during pregnancy at another center but delivered in our hospital could not be detected. Fourth, although being a matched case control study describing non-obstetric surgical procedures during pregnancy in a single center, the number of cases remains small. Consequently, the study may be underpowered for certain outcomes. However, even in a recent nationwide population study, the number of cases was only 2.7 times higher and included $33 \%$ obstetric surgeries. ${ }^{8}$ Fifth, we could only make a comparison between exact birth weights in the matched-controlled analysis, because data for the entire group were only available as categorical data ( $>$ and $<2500 \mathrm{~g}$ ). Sixth, caution is warranted when interpreting causality. As mentioned, this retrospective study reports associations (for example between anesthesia and pregnancy outcomes) rather than proving causality. Finally, while also affecting low-risk obstetric services, our hospital population consists of a mixture of low- and high-risk patients, with over-representation of high risk patients compared to the general obstetric population. This also makes our control population intrinsically more prone to adverse pregnancy outcomes.

In conclusion, women undergoing invasive procedures during pregnancy delivered earlier and preterm more frequently. Although mean birth weight was lower in the interventional group, the frequency of low birth weight babies did not increase after non-obstetric surgery during pregnancy. Intra-abdominal surgery $(79 \%$ laparoscopic techniques) was associated with similar outcomes to extra-abdominal surgery. The use of GA was associated with a higher incidence of low birth weight babies. It is unknown whether this should be attributed to the intervention, the anesthesia or the underlying surgical condition. Interventions during the third trimester had the highest risk for preterm birth within two weeks of the operation.

\section{Funding}

The authors did not receive any specific grant from funding agencies in the public, commercial, or not-forprofit sectors.

\section{References}

1. Reitman E, Flood P. Anaesthetic considerations for non-obstetric surgery during pregnancy. Br J Anaesth 2011;107(suppl 1):i72-8.
2. Mazze RI, Källén B. Reproductive outcome after anesthesia and operation during pregnancy: a registry study of 5405 cases. Am J Obstet Gynecol 1989;161:1178-85.

3. Jenkins TM, Mackey SF, Benzoni EM, Tolosa JE, Sciscione AC. Non-obstetric surgery during gestation: risk factors for lower birthweight. Aust N Z J Obstet Gynaecol 2003;43:27-31.

4. Cohen-Kerem R, Railton C, Oren D, Lishner M, Koren G. Pregnancy outcome following non-obstetric surgical intervention. Am J Surg 2005;190:467-73.

5. Balinskaite V, Bottle A, Sodhi V, et al. The risk of adverse pregnancy outcomes following nonobstetric surgery during pregnancy: estimates from a retrospective cohort study of 6.5 million pregnancies. Ann Surg 2017;266:260-6.

6. Yi Wen P, Broom E, Flatley C, Kumar S. Maternal demographic and intrapartum antecedents of severe neonatal outcomes at term. J Matern Fetal Neonatal Med 2017;Nov8;1-6. https://doi.org/ 10.1080/14767058.2018.1540581.

7. Goodman S. Anesthesia for nonobstetric surgery in the pregnant patient. Semin Perinatol 2002;26:136-45.

8. Yu C-H, Weng S-F, Ho C-H, et al. Pregnancy outcomes following nonobstetric surgery during gestation: a nationwide populationbased case-control study in Taiwan. BMC Pregnancy Childbirth 2018;18:460.

9. FDA Drug Safety Communication: FDA review results in new warnings about using general anesthetics and sedation drugs in young children and pregnant women. http://www.fda.gov/downloads/Drugs/DrugSafety/UCM533197.pdf. Accessed December 20, 2018 .

10. Palumbi R, Peschechera A, Margari M, et al. Neurodevelopmental and emotional-behavioral outcomes in late-preterm infants: an observational descriptive case study. BMC Pediatr 2018;18:318.

11. Fitzgerald E, Boardman JP, Drake AJ. Preterm birth and the risk of neurodevelopmental disorders - is there a role for epigenetic dysregulation? Curr Genomics 2018;19:507-21.

12. Schwarzman P, Baumfeld Y, Bar-Niv Z, et al. The effect of nonobstetric invasive procedures during pregnancy on perinatal outcomes. Arch Gynecol Obstet 2015;292:603-8.

13. Abbasi N, Patenaude V, Abenhaim HA. Evaluation of obstetric and fetal outcomes in pregnancies complicated by acute appendicitis. Arch Gynecol Obstet 2014;290:661-7.

14. Vannevel V, Jans G, Bialecka M, et al. Internal herniation in pregnancy after gastric bypass. Obstet Gynecol 2016;127:1013-20.

15. Visser BC, Glasgow RE, Mulvihill KK, Mulvihill SJ. Safety and timing of nonobstetric abdominal surgery in pregnancy. Dig Surg 2001; 18:409-17.

16. Chung JC, Cho GS, Shin EJ, Kim HC, Song OP. Clinical outcomes compared between laparoscopic and open appendectomy in pregnant women. Can J Surg 2013;56:341-6.

17. Kwon H, Lee M, Park HS, et al. Laparoscopic management is feasible for nonobstetric surgical disease in all trimesters of pregnancy. Surg Endosc 2018;32:2643-9.

18. Walters JL, Paule MG. Review of preclinical studies on pediatric general anesthesia-induced developmental neurotoxicity. Neurotoxicol Teratol 2017;60:2-23.

19. Devroe S, Lemiere J, Van Hese L, et al. The effect of xenonaugmented sevoflurane anesthesia on intraoperative hemodynamics and early postoperative neurocognitive function in children undergoing cardiac catheterization: a randomized controlled pilot trial. Paediatr Anaesth 2018;28:726-38.

20. Chevrot A, Kayem G, Coupaye M, et al. Impact of bariatric surgery on fetal growth restriction: experience of a perinatal and bariatric surgery center. Am J Obstet Gynecol 2016;214(655):e1-7.

21. Andropoulos DB, Greene MF. Anesthesia and developing brains Implications of the FDA warning. $N$ Engl $J$ Med 2017;376:905-7. 